University of Nebraska - Lincoln

DigitalCommons@University of Nebraska - Lincoln

June 1967

\title{
Inelastic Collisions of the Second Kind between Electrons and Excited Mercury Atoms
}

Paul Burrow
University of Nebraska - Lincoln, pburrow1@unl.edu

Follow this and additional works at: https://digitalcommons.unl.edu/physicsburrow

Part of the Physics Commons

Burrow, Paul, "Inelastic Collisions of the Second Kind between Electrons and Excited Mercury Atoms" (1967). Paul Burrow Publications. 30.

https://digitalcommons.unl.edu/physicsburrow/30

This Article is brought to you for free and open access by the Research Papers in Physics and Astronomy at DigitalCommons@University of Nebraska - Lincoln. It has been accepted for inclusion in Paul Burrow Publications by an authorized administrator of DigitalCommons@University of Nebraska - Lincoln. 
discrepancy between the measurements and calculations seem likely.

Similarly, appearance potential data are consistent with the assumption that only a minor fraction of the ions come from excited states which were not considered in the calculations. Two other pieces of evidence also substantiate this conclusion. First, the angular distribution $^{1}$ of ions over the entire range of proton energies observed is characteristic of a $1 s \sigma_{g} \rightarrow 2 p \sigma_{u}$ transition. Second, optical observation ${ }^{8}$ of the excited ionic states show the cross section to be too small to account for more than a few percent of the observed ions.

The temperature of the collision chamber was measured and was low enough that rotational excitation should not have contributed significantly-particularly in view of the small effects produced by such excitation. At these temperatures vibrational excitation should be

${ }^{8}$ E. R. Williams, J. V. Martinez, and G. H. Dunn, Bull. Am. Phys. Soc. 12, 233 (1967). entirely negligible and thus is not considered as a possible explanation.

Deficiencies in the calculation may lie in the FranckCondon assumption-namely that the electronic matrix element is nearly independent of $R$ over the range of $R$ where $\chi_{v}(R)$ has appreciable values. Strong variations in such electronic factors are known for other transitions, ${ }^{9-12}$ but no examples are available which would produce the large discrepancies observed here.

One further possibility is that repulsive high-lying Rydberg states of $\mathrm{H}_{2}$ autoionize to form the observed $\mathrm{H}^{+}$with apparent appearance potential consistent with the $2 p \sigma_{u}$ state of $\mathrm{H}_{2}{ }^{+}$.

We conclude that these latter two possibilities are the most likely explanations of the observed discrepancy.

\footnotetext{
${ }^{9}$ Gordon H. Dunn and Bert Van Zyl, Phys. Rev. 154, 40 (1967).

${ }_{10} \mathrm{~J}$. M. Peek, Phys. Rev. 154, 52 (1967); 134, A877 (1964); 140, A11 (1965).

11 D. R. Bates, J. Chem. Phys. 19, 1122 (1951).

${ }_{12}$ M. R. Flannery and V. Öpik, Proc. Phys. Soc. (London) 86, 491 (1965).
}

\title{
Inelastic Collisions of the Second Kind between Electrons and Excited Mercury Atoms*
}

\author{
P. D. Burrow $\dagger$ \\ University of California, Berkeley, California
}

(Received 12 January 1967)

\begin{abstract}
The relative cross section for inelastic collisions of the second kind between electrons and mercury atoms in the excited ${ }^{3} P_{1}$ state was measured as a function of electron energy. Excited atoms were produced by absorption of the $2537 \AA$ resonance line from a mercury arc. A low-energy beam of electrons was produced using the retarding potential difference method. Scattered electrons which gained kinetic energy were detected by passing them through a potential barrier which reflected unscattered electrons. The data are in good agreement with the cross section computed by applying detailed balancing to the known cross section for excitation to the ${ }^{3} P_{1}$ state by electron impact. Information about the angular scattering dependence was also obtained.
\end{abstract}

\section{INTRODUCTION}

$\mathrm{D}$ IRECT measurement of inelastic collisions of the second kind between electrons and excited atoms has been attempted in only a few early studies. ${ }^{1-4}$ The superelastic collision reported in this paper is shown in Eq. (1):

$$
e+\mathrm{Hg}\left({ }^{3} P_{1}\right) \rightarrow \mathrm{Hg}\left({ }^{1} S_{0}\right)+e+4.89 \mathrm{eV} .
$$

An electron striking an excited mercury atom is scattered with increased kinetic energy leaving the atom in its ground state.

\footnotetext{
* Research supported by the U. S. Office of Naval Research.

$\dagger$ Present address: Yale University, Department of Engineering and Applied Sciences, New Haven, Connecticut.

${ }^{1}$ H. D. Smyth, Proc. Natl. Acad. Sci. (U. S.) 11, 679 (1925)

2 H. D. Smyth, Phys. Rev. 22, 108 (1926).

3 A. Leipunsky and E. Strauff, Z. Physik 58, 104 (1929).

${ }^{4}$ G. D. Latyscheff and A. I. Leipunsky, Z. Physik 65, 111 (1930)
}

Smyth ${ }^{1}$ attempted to measure the energy given to one electron during dielectronic formation of $\mathrm{I}^{-}$. Smyth, ${ }^{2}$ and Leipunsky and Strauff ${ }^{3}$ tried to measure superelastic electrons from excited states produced by chemical reactions. Latyscheff and Leipunsky ${ }^{4}$ measured fast electrons from excited states in mercury vapor which were populated by absorption of the 2537 - $\AA$ resonance line. Their results were not clear-cut because of the primitive techniques of the time. In addition, they assumed that most of the excited atoms were in the ${ }^{3} P_{0}$ metastable state as a result of de-excitation from the ${ }^{3} P_{1}$ state by collision with normal atoms. This process is unlikely at the pressures they used.

The present work was undertaken to produce superelastic collisions with a known excited state of mercury and to study the dependence of the relative cross section on energy. 


\section{EXPERIMENT}

\section{A. Description of Tube}

A diagram of the tube used in this experiment is shown in Fig. 1. Plates $S_{1}$ through $S_{5}$ form an electron gun which can be operated in the retarding potential difference $^{5}$ mode to improve the energy resolution. Plates $S_{5}$ and $S_{6}$ form a field-free collision chamber. Plate $S_{7}$ is a retarding electrode used to analyze the energy of the main beam and the scattered electrons. Current arriving at the electron collector EC is measured by a vibrating-reed electrometer. The electron beam is collimated by a uniform magnetic field of $180 \mathrm{G}$ produced by a Helmholz coil.

Mercury atoms in the excited ${ }^{3} P_{1}$ state are produced in the collision chamber by absorption of the $2537-\AA$ resonance line from an external mercury arc. To admit the ultraviolet light, the chamber is surrounded by a concentric quartz cylinder. To make the tube vacuumtight, the ends of the cylinder were polished and attached to two stainless-steel endplates by means of epoxy. The endplates support the electrodes and the vacuum tubulation.

All metal parts of the gun are constructed of nonmagnetic stainless steel and were fired in vacuum before assembly to reduce outgassing. The tube was baked at $90^{\circ} \mathrm{C}$ for $10 \mathrm{~h}$ and reached a background pressure of $2 \times 10^{-7}$ Torr. To guarantee that impurities are kept at a low level, the tube is pumped on continuously at low speed.

The mercury vapor pressure in the collision chamber is varied by controlling the temperature of a side tube containing liquid mercury. The tube itself is maintained at a constant temperature of $50^{\circ} \mathrm{C}$.

\section{B. The Mercury Arc}

A mercury arc, similar to that described by Houtermans, ${ }^{6}$ provides a strong, unreversed source of $2537-\AA \AA$ resonance radiation. The arc is toroidal in shape to allow light to enter the collision chamber from a large solid angle.

Fluctuations in the light intensity can cause considerable noise because of the photoelectrons liberated from the metal electrodes. Most of this current is shielded from the collector. However, photons absorbed and re-radiated in the collision chamber may eject electrons from the slit edges, causing a background current which fluctuates with the light intensity. The stability of the arc described here is excellent. The peak-to-peak noise due to the photocurrent is $6 \times 10^{-16} \mathrm{~A}$ when using a 1-sec time constant in the electrometer. Attempts to use low pressure arcs with mercury pool electrodes, both with and without cathode spot fixers, were unsuccessful because of large light fluctuations.

${ }^{5}$ R. E. Fox, W. M. Hickam, D. J. Grove, and T. Kjeldaas, Jr., Rev. Sci. Instr. 26, 1101 (1955).

${ }^{6}$ F. G. Houtermans, Z. Physik 76, 474 (1932).

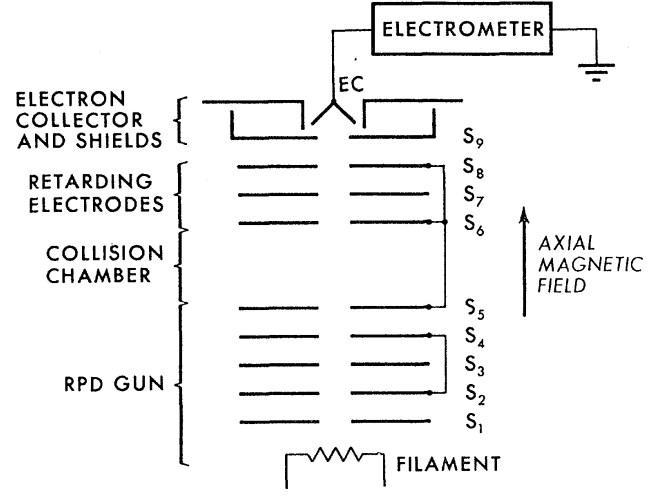

FIG. 1. Diagram of the experimental tube.

\section{RESULTS}

\section{A. Retarding Curve}

Consider now a beam of low-energy electrons sent into the collision chamber. With atoms in excited states present, the current which arrives at the electron collector, with no retarding voltage at $S_{7}$, consists of unscattered, elastically scattered, and superelastically scattered electrons. By increasing the retarding voltage, the main beam can be entirely reflected, allowing only the superelastic electrons to arrive at the collector.

A typical retarding curve showing electron collector current as a function of the retarding voltage is shown in Fig. 2. The left side shows the main electron beam, having $0.8 \mathrm{eV}$ of kinetic energy, being retarded. The voltage is measured with respect to the filament and is corrected for contact potential shift by requiring the position of maximum slope to be at zero voltage. At retarding voltages of $-2.8 \mathrm{~V}$, well beyond the highenergy tail of the main beam, the small signal plotted with open circles could be seen. As the retarding voltage is made more negative, the signal gradually decreases into the noise somewhat above $-5.1 \mathrm{~V}$. If superelastic collisions occur with atoms in the ${ }^{3} P_{1}$ state, then $4.89 \mathrm{eV}$ is the maximum energy that electrons can gain. Considering the small signal and the spread in energy of the main beam, which is $0.3 \mathrm{eV}$ at halfmaximum for this curve, the data are in fair agreement.

The signal could not be measured for retarding voltages between zero and $-2.8 \mathrm{~V}$. In this region, a portion of the high-energy tail of the main beam is collected and there is considerable scatter in the data.

The gradual decline in scattered current as a function of increasingly negative retarding voltage is a result of the angular dependence of the scattering process. The main electron beam is retarded abruptly, that is, within a few tenths of an electron volt, because essentially all of the electron velocity is directed along the axis of the tube. The scattered electrons, however, are distributed as a function of the angle from the tube axis. Because of the magnetic field, the electrons cannot 


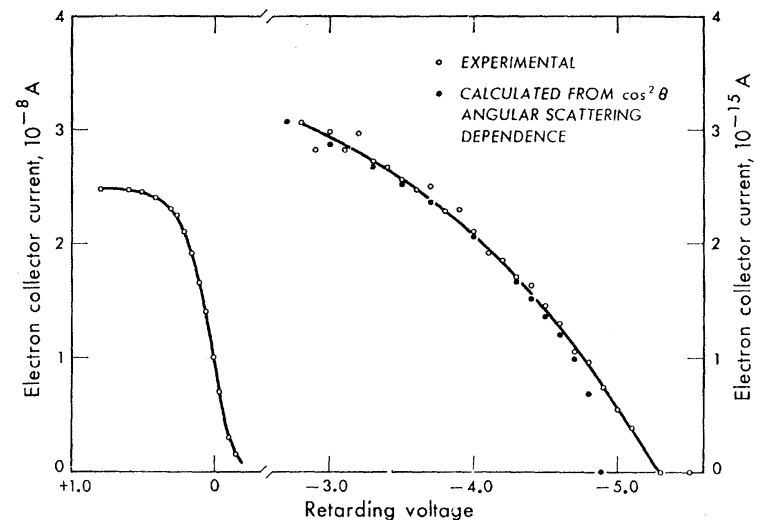

Frg. 2. The electron collector current as a function of the retarding voltage at plate $S_{7}$. The electron beam has an energy of $0.8 \mathrm{eV}$. The closed circles are calculated by assuming the electrons are scattered with a $\cos ^{2} \theta$ dependence. This curve is normalized to the experimental data at $-4.0 \mathrm{~V}$.

escape and are forced to spiral down the axis. The retarding field acts only against the component of velocity parallel to the axis. Therefore, electrons scattered at large angles are reflected with less retarding voltage. The range of voltage plotted in Fig. 2 corresponds to electrons scattered between zero and $40^{\circ}$ from the axis.

\section{B. Relative Cross Section}

If now the accelerating voltage of the main beam is varied keeping the retarding voltage adjusted to the same position, say $-3.2 \mathrm{~V}$, on the retarding curve, the relative cross section as a function of energy may be found. The scattered current must be normalized at each energy by the magnitude of the main beam, which must be measured necessarily without retardation. It is assumed that the path length traversed by the reflected beam does not vary with energy. The effect of a changing elastic cross section is discussed in Sec. III C.

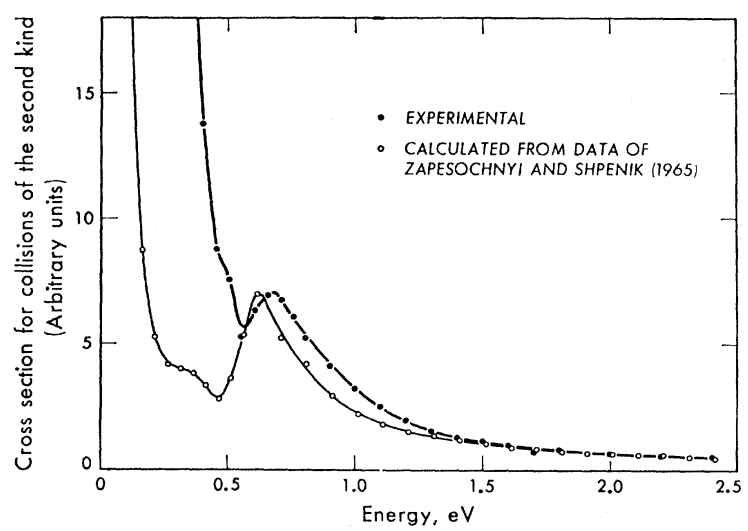

Fig. 3. Comparison of cross sections for collisions of the second kind as a function of electron energy. The curve plotted with open circles is calculated from the cross section for collisions of the first kind by using detailed balancing and is normalized to the experimental data at $2.0 \mathrm{eV}$.
The closed circles in Fig. 3 show the superelastic cross section for electrons scattered between zero and $35^{\circ}$ from the axis. ${ }^{7}$ The region below $0.9 \mathrm{eV}$ is taken with an electron energy spread of $0.2 \mathrm{eV}$ at half-maximum. Above this, the cross section is much smaller and it is necessary to broaden the beam to get a detectable signal. Although the cross section is quite large between zero and $0.3 \mathrm{eV}$, the electron beam current drops off very rapidly as the energy is decreased and it is not possible to see a signal below $0.3 \mathrm{eV}$. The data shown in Fig. 3 represent the best run that was taken. Other data show more scatter or inferior resolution but preserve the general features.

\section{Pressure Variation}

The data shown in Figs. 2 and 3 were taken at mercury pressures in the low $10^{-3}$ Torr range. At lower pressures, the density of excited states is too small to

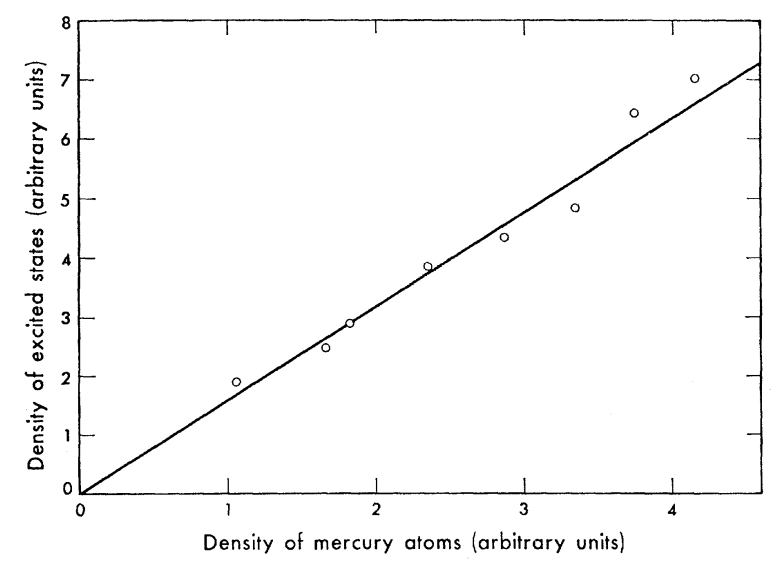

FIG. 4. Density of excited states as a function of mercury density.

produce a detectable signal; at higher pressure, the main electron beam is appreciably attenuated because of elastic scattering by atoms in the ground state.

Although the mercury pressure is not known absolutely because of the low-speed pumping on the tube to remove impurities, the relative pressure may be varied by controlling the mercury reservoir temperature. The density of the excited states, which is proportional to the scattered current normalized by the beam current, at any given energy, is plotted as a function of the relative mercury density in Fig. 4. This curve was taken at an electron energy of $0.8 \mathrm{eV}$. The linearity indicates that the mercury vapor is optically thin. At higher densities, most of the absorption of the 2537-A line would take place near the walls and the density of excited states in the center of the tube would decrease. The linear relationship also indicates that no change

${ }^{7}$ Because the main beam is reflected, the superelastic current may also contain a contribution from electrons backscattered into angles from $145^{\circ}$ to $180^{\circ}$. 
in electron path length has occurred because of increased elastic scattering in the range of densities used. This implies that the path length would also be unaffected by a similar sized change in the elastic cross section with energy.

\section{DISCUSSION}

\section{A. Angular Scattering Dependence}

Since the initial state of a mercury atom before a superelastic collision is the ${ }^{3} P_{1}$ state and the final state is the ${ }^{1} S_{0}$ ground state, the electron gaining energy must also gain one unit of angular momentum. In the limit of zero velocity for the incoming electrons, that is, at the threshold for the superelastic collision process, the electron beam carries no angular momentum. After the collision, conservation of angular momentum requires that the scattered electrons each have one unit of angular momentum. In terms of a partial-wave analysis, the only component of the scattered current is the $l=1$ or $p$ wave. This argument is rigorously true only at threshold. However, for low energies we may expect the $p$ wave to be the most important component of the scattered current in the absence of resonance effects.

This partial wave has an angular scattering probability which varies as $\cos ^{2} \theta$. By finding the axial component of the velocity corresponding to scattering at angle $\theta$, it is simple to compute the retarding curve, which is plotted in closed circles in Fig. 2. The curve is normalized to the experimental data at $-4.0 \mathrm{~V}$. The agreement with the data taken at a beam energy of $0.8 \mathrm{eV}$ is good.

By taking retarding curves for many beam voltages, the angular scattering could be studied as a function of energy. The signal and resolution are not good enough to warrant this except at the energy used here, where the signal is largest.

\section{B. Detailed Balancing}

Cross sections for collisions of the first and second kind are related by the principle of detailed balancing. Klein and Rosseland ${ }^{8}$ first predicted the existence of superelastic collisions from this consideration and derived the relationship shown in Eq. (2). Let $\sigma_{12}$ and $\sigma_{21}$ be the cross sections for collisions of the first and second kind, respectively, let $\Delta E$ be the energy difference between the two states of the atom, and $g_{1}$ and $g_{2}$ be the degeneracies of the lower and upper atomic states, respectively:

$$
\sigma_{21}(E)=\left(g_{1} / g_{2}\right)((E+\Delta E) / E) \sigma_{12}(E+\Delta E) .
$$

Fortunately, data concerning the relative cross section for collisions of the first kind in mercury have been

${ }^{8}$ O. Klein and S. Rosseland, Z. Physik 4, 46 (1921).

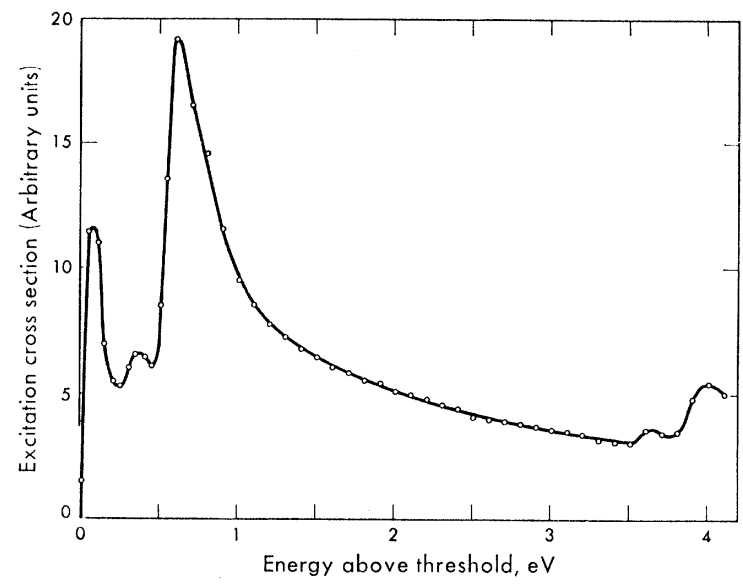

Fig. 5. The relative cross section for excitation to the ${ }^{3} P_{1}$ state of mercury by electron impact as measured by Zapesochnyi and Shpenik. The curve is shifted by $4.89 \mathrm{eV}$.

recently published. ${ }^{9,10}$ The superelastic cross section is easily calculated from this by the Klein-Rosseland equation above and offers a check on the direct measurement made here. Since the structure in the excitation function is directly related to that in the superelastic cross section, a discussion of several interesting features follows.

\section{Collisions of the First Kind}

Zapesochnyi and Shpenik ${ }^{9,10}$ have measured the excitation function of the 2537-Å line using an electrostatic analyzer to produce an electron beam with a halfwidth of less than $0.1 \mathrm{eV}$. Figure 5 shows this excitation function which has been shifted for convenience so that the threshold, which is at $4.89 \mathrm{eV}$, occurs at zero energy. This curve, which should not be used as a primary reference, was taken from a photographic enlargement of their curve, which was very compactly plotted.

Just above threshold, an extremely sharp peak was found which coincides in energy with sharp resonance structure found in the total scattering of electrons from mercury atoms investigated by Kuyatt, Simpson, and Mielczarek. ${ }^{11}$ This structure is attributed by Fano and Cooper $^{12}$ to the formation of a temporary negative ion in the $\left(6 s 6 p^{2}\right)^{4} P_{5 / 2}$ state. This short-lived ion can decay leaving the atom in the ${ }^{3} P_{1}$ state. The sharp peak in the excitation function appears to be due to this additional means of production.

The excitation function also contains a small peak at $0.35 \mathrm{eV}$ above threshold. Although no well-resolved

${ }^{9}$ I. P. Zapesochnyi and O. B. Shpenik, Dokl. Akad. Nauk SSSR 160, 1053 (1965) [English transl.: Soviet Phys.-Doklady 10, 140 (1965)].

${ }^{10}$ I. P. Zapesochnyi and O. B. Shpenik, Zh. Eksperim. i Teor. Fiz. 50, 890 (1966) [English transl.: Soviet Phys.-JETP 23, $592(1966)]$.

11 C. E. Kuyatt, J. Arol Simpson, and S. R. Mielczarek, Phys. Rev. 138, A385 (1965).

12 U. Fano and J. W. Cooper, Phys. Rev. 138, A400 (1965). 
resonance was listed here by Kuyatt, Simpson, and Mielczarek, ${ }^{11}$ it is suggested that this peak is due to one of the higher states of the $6 s 6 p^{2}$ configuration, such as the ${ }^{2} D,{ }^{2} S$, or ${ }^{2} P$, which decays leaving the atom in the ${ }^{3} P_{1}$ state.

The main peak at $0.6 \mathrm{eV}$ occurs just above the threshold for excitation to the metastable ${ }^{3} P_{2}$ state. Zapesochnyi and Shpenik ${ }^{10}$ suggest that this peak results from a quantum-mechanical interaction between the ${ }^{3} P_{1}$ and ${ }^{3} P_{2}$ states. Although the width of the peak is greater than that of the resonance peak at threshold, the low-energy side is quite steep, rising to a maximum in $0.1 \mathrm{eV}$. This suggests a resonance process. Again, it is plausible that higher temporary negative ion states near the metastable threshold could provide the mechanism.

Finally, the peaks higher than $3 \mathrm{eV}$ above threshold are due to excitation to levels which cascade to the ${ }^{3} P_{1}$ state. The direct part of the excitation is not easily separated above this energy.

\section{Comparison}

Using the Klein-Rosseland equation given above, the cross section for collisions of the second kind computed from the data of Zapesochnyi and Shpenik is shown in Fig. 3. It is normalized to the experimental data found here at $2 \mathrm{eV}$. The sharp peak just above threshold is now greatly expanded by the rapidly rising multiplier $(E+\Delta E) / E$ as $E$ approaches zero, and is not included in the graph.

The principal features of the curves are the same and agreement is good except near the resonance peak just above threshold. The differences arise from two sources. First, the energy spread of the electron beam used here is two to three times larger than that used by Zapeso-

\footnotetext{
${ }^{13} \mathrm{I}$ am indebted to Dr. P. J. MacVicar-Whelan for writing the computer program for this calculation.
}

chnyi and Shpenik. To assess the effect of energy spread, a computer was used to simulate the measurement of the high resolution data with the electron energy distribution used here. ${ }^{13}$ Although closer agreement is found, there remains a disparity at low energies.

The second source of disagreement is connected with the energy dependence of the angular scattering. The function calculated from Zapesochnyi and Shpenik's data is a total cross section for electrons scattered at all angles. The cross section measured here includes only those electrons scattered between zero and $35^{\circ}$. If the angular scattering dependence changes with energy, then the fraction of scattered electrons which is collected also changes and these two curves need not agree. The most likely region for this to happen is near the resonance structure.

If we assume that the negative ion has the configuration suggested by Fano and Cooper, ${ }^{12}$ then for the peak near threshold we have the following reaction:

$e+\mathrm{Hg}\left({ }^{3} P_{1}\right) \rightarrow \mathrm{Hg}\left({ }^{4} P_{5 / 2}\right) \rightarrow \mathrm{Hg}\left({ }^{1} S_{0}\right)+e+4.89 \mathrm{eV}$.

Conserving both total angular momentum and parity requires the scattered electron to leave with two units of angular momentum. This wave is strongly peaked in the forward direction, which implies that the fraction of the scattered electrons collected in the resonance region is larger than that collected at other energies. This causes the cross section to appear larger relative to that at higher energies and agrees qualitatively with the data measured here.

\section{ACKNOWLEDGMENTS}

I wish to thank Professor Leonard B. Loeb, under whom this work was carried out, for his support and encouragement; Dr. J. T. Dowell, for suggesting the topic; and Dr. Dowell, Dr. D. A. MacLennan, and Dr. P. J. MacVicar-Whelan, for useful discussions. 